\title{
El arte como instrumento para desarrollar el espíritu crítico
} Art as an instrument to develop a critical approach

Continuamos con la serie de artículos relacionados con el arte como instrumento educativo en medicina, basada en la propuesta de utilizar el lenguaje simbólico del arte como recurso docente en la formación de los profesionales de la salud. ${ }^{1}$

En esta oportunidad analizaremos la naturaleza y utilidad del espíritu crítico a la luz de un poema del genial escritor Fernando Pessoa, a través de su heterónimo Alexander Search, en las estrofas de su poema "Manía de la Duda":2

"Todas las cosas son un enigma para mí Que nace de repente de las mismas evidencias

Las cosas son y parecen, y nada nos revela

El secreto de la vida que la apariencia oculta Al descubrir

Que cuanto más claras las cosas se revelan Mayor misterio dentro de sí ocultan"

El espíritu crítico consiste en la actitud de evaluar finamente no sólo la labor ajena sino especialmente la propia, siendo autoexigentes y perseverantes a la hora de dar lo mejor de nosotros. ${ }^{3}$ Resulta entonces que cultivar dicha actitud se traduce, como veremos a continuación, en beneficios tanto para el paciente como para el médico.

En un artículo anterior ("El arte como instrumento para comprender la diferencia entre el enfermo y la enfermedad"), analizamos la diferencia existente entre el proceso fisiopatológico que afecta a un individuo (el enfermo) y la construcción teórica que se elabora para su abordaje interpretativo (la enfermedad). ${ }^{4}$ Es precisamente el espíritu crítico del médico, mediante el replanteo continuo acerca de la exactitud de sus interpretaciones, quien reduce el margen de error en las mismas, aproximando así sus diagnósticos a la realidad, y por ende sus prescripciones al éxito terapéutico.
Por otra parte, el médico que valora el potencial de mejora que encierra el reconocimiento de la ignorancia y del error (propio o ajeno), aprende a pensar críticamente, a aprender y desaprender (Alvin Toffler), y transita de esta manera la única senda posible hacia la transformación y el crecimiento profesional y espiritual. ${ }^{5,6}$

Finalmente, no debe olvidarse que la curiosa ignorancia (James Clerk Maxwell) es motor de la búsqueda y adquisición del conocimiento, el cuál en definitiva consiste en la obtención de respuestas que conducen a nuevas preguntas, es decir de la ignorancia a una docta ignorancia (Nicolás de Cusa). ${ }^{7,8}$

Concluimos entonces que la adquisición de un espíritu crítico resulta fundamental para un óptimo ejercicio y desarrollo médico y personal, y que el arte puede llegar a ser un valioso recurso a tal fin.

Dr. Carlos G. Musso y Dra. Paula A. Enz Escuela de Medicina, Instituto Universitario del Hospital Italiano de Buenos Aires - Argentina

http:/ /dx.doi.org/10.5546/aap.2016.100

\section{REFERENCIAS}

1. Musso CG, Enz PA. El arte como instrumento educativo en medicina. Arch Argent Pediatr 2014;112(6):494-5.

2. Pessoa F. Máscaras y paradojas. Barcelona: Eldhasa; 1996.

3. Ceriani Cernadas JM. La actitud crítica en medicina: cada vez más necesaria. Arch Argent Pediatr 2012;110(3):194-5.

4. Musso CG, Enz PA. El arte como instrumento para comprender la diferencia entre el enfermo y la enfermedad. Arch Argent Pediatr 2015;113(4):292-3.

5. Bartels D. Las buenas preguntas: No es en el aula donde mejor se aprende a pensar de forma crítica. Investigación y ciencia 2013;444:56.

6. Jung C. El Libro Rojo. Buenos Aires: El hilo de Ariadna, 2012.

7. Palma H. Filosofía de la ciencia. San Martin: UNSAM. 2010.

8. Firestein S. Ignorance: How it drives Science. Oxford. Oxford University Press 2012. 